Annales Geophysicae (2002) 20: 947-951 C European Geophysical Society 2002

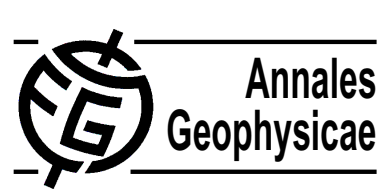

\title{
Neural network prediction of relativistic electrons at geosynchronous orbit during the storm recovery phase: effects of recurring substorms
}

\author{
M. Fukata ${ }^{1}$, S. Taguchi ${ }^{1}$, T. Okuzawa ${ }^{1}$, and T. Obara ${ }^{2}$ \\ ${ }^{1}$ Dept. of Information and Communication Engineering, University of Electro-Communications, Chofu, 182-8585, Japan \\ ${ }^{2}$ Communications Research Laboratory, Koganei, 184-8795, Japan
}

Received: 1 October 2001 - Revised: 5 March 2002 - Accepted: 30 April 2002

\begin{abstract}
During the recovery phase of geomagnetic storms, the flux of relativistic $(>2 \mathrm{MeV}$ ) electrons at geosynchronous orbits is enhanced. This enhancement reaches a level that can cause devastating damage to instruments on satellites. To predict these temporal variations, we have developed neural network models that predict the flux for the period $1-12 \mathrm{~h}$ ahead. The electron-flux data obtained during storms, from the Space Environment Monitor on board a Geostationary Meteorological Satellite, were used to construct the model. Various combinations of the input parameters $A L, \Sigma A L$, $D_{s t}$ and $\Sigma D_{s t}$ were tested (where $\Sigma$ denotes the summation from the time of the minimum $D_{s t}$ ). It was found that the model, including $\Sigma A L$ as one of the input parameters, can provide some measure of relativistic electron-flux prediction at geosynchronous orbit during the recovery phase. We suggest from this result that the relativistic electron-flux enhancement during the recovery phase is associated with recurring substorms after $D_{s t}$ minimum and their accumulation effect.
\end{abstract}

Key words. Magnetospheric physics (energetic particles, trapped; magnetospheric configuration and dynamics; storms and substorms)

\section{Introduction}

During the recovery phase of geomagnetic storms, the flux of relativistic $(>2 \mathrm{MeV})$ electrons at geostationary orbits (GEO) is enhanced. This enhancement often reaches a level that is higher than the prestorm value (e.g. Nagai, 1988; Baker et al., 1997; Obara et al., 2000a). This enhancement is not accounted for by the flux of energetic electrons in the interplanetary medium alone ( $\mathrm{Li}$ et al., 1997). This implies that some energization or transportation process operates on the energetic electrons in the magnetosphere. Although the

Correspondence to: S. Taguchi (taguchi@ice.uec.ac.jp) mechanism for this process has been suggested by several researchers (e.g. Freeman et al., 1998; Nakamura et al., 1998; Obara et al., 2000b), there is still considerable uncertainty about the details.

High flux levels of relativistic electrons can cause irreparable damage to the instruments on satellites (Gussenhoven et al., 1991; Baker et al., 1997), and this practical consequence of the flux enhancement has promoted the development of empirical models. Nagai (1988) designed a linear prediction filter for the prediction of daily averages of electron flux at GEO, using the $K_{p}$ index as an input. This model predicted successfully the electron flux on a daily scale. Koons and Gorney (1991) also made predictions of the daily average flux at GEO using artificial neural networks (ANN).

Modeling using ANN has predicted successfully the energetic-electron flux with a time resolution of $1 \mathrm{~h}$ (Stringer et al., 1996; Freeman et al., 1998). Although the energy range of the electrons for these two models is different (3$5 \mathrm{MeV}$ for Stringer et al., 1996 and $100 \mathrm{keV}$ to $1.5 \mathrm{MeV}$ for Freeman et al., 1998), both models used the $D_{s t}$ index, and the electron flux as input data. Stringer et al. (1996) used the current flux of energetic electrons $(3-5 \mathrm{MeV})$, and those which existed 1 to $3 \mathrm{~h}$ earlier. Freeman et al. (1998) used the current low-energy $(35 \mathrm{keV})$ electron flux, plus those existing $15 \mathrm{~min}$ and $75 \mathrm{~min}$ earlier, to predict the energetic $(100 \mathrm{keV}$ to $1.5 \mathrm{MeV})$ electron flux for the storm of 3-4 November 1993. It would appear that the high performance of these models is due to the inclusion of the variation of electrons existing earlier as data. Since the use of the model has important practical applications, it not only needs to help us understand the physical mechanism, but also must use widely available input parameters. In this paper, we show ANN modelling of the relativistic-electron flux for the recovery phase is possible using $\Sigma A L$ (the summation of $A L$ from the time of $D_{s t}$ minimum in the main phase), and we suggest the significance of recurring substorms in the flux enhancement. 

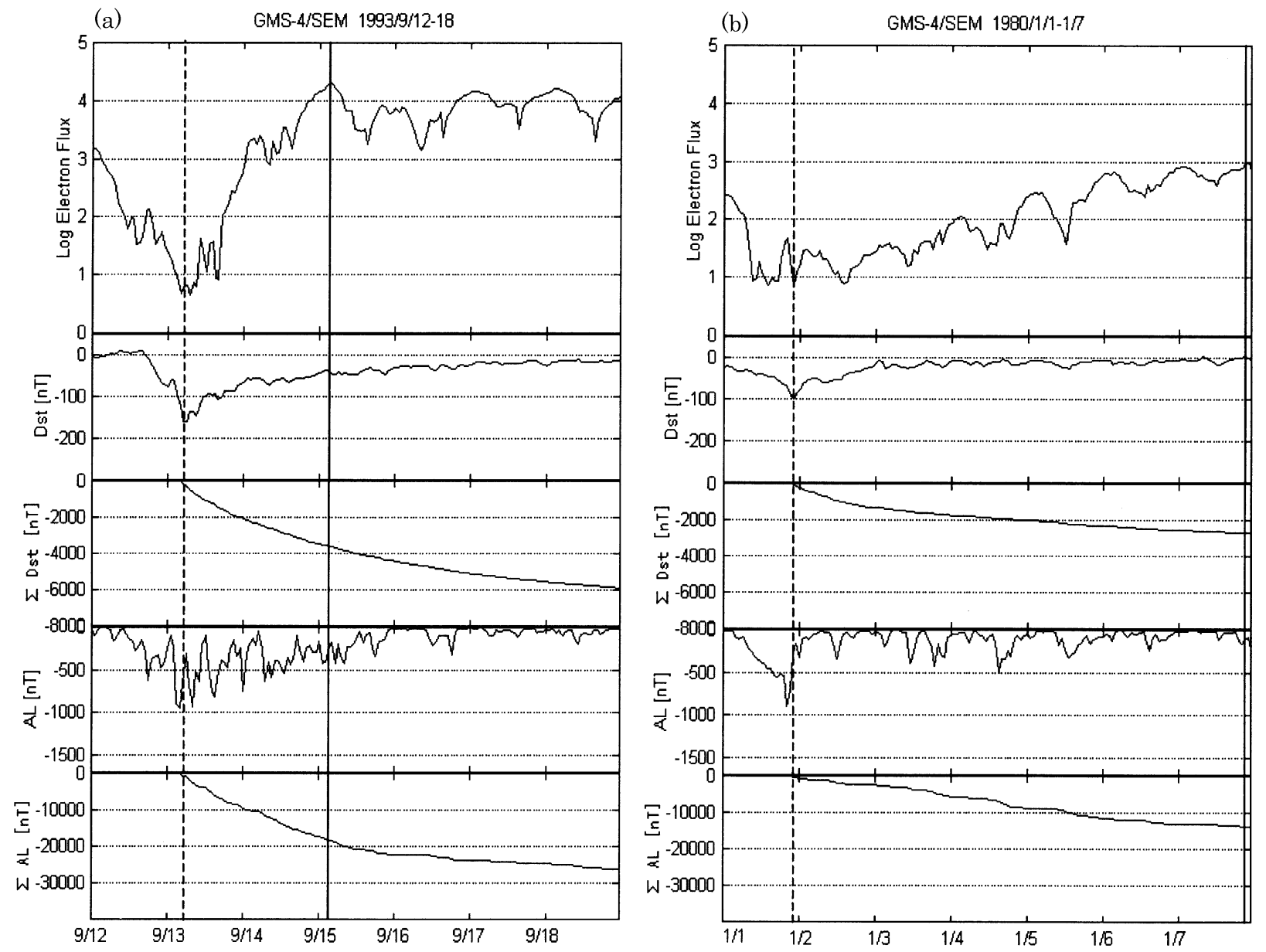

Fig. 1. Examples of enhancements of relativistic ( $>2 \mathrm{MeV}$ ) electron flux observed by Geostationary Meteorological Satellite and $D_{s t} / A L$ indices (a) for the the storm of 12-18 September 1993, and (b) for the storm of 1-7 January 1980. $\Sigma D_{s t}$ and $\Sigma A L$ are also plotted, where $\Sigma$ denotes the summation from the time of the minimum $D_{s t}$. The minimum $D_{s t}$ is represented with a dashed vertical line, and a solid line represents the time for the peak electron flux for the six-day period after the $D_{s t}$ minimum.

\section{$2 \quad A L$ index as model input}

We used data for relativistic $(>2 \mathrm{MeV})$ electrons that were obtained from the Space Environment Monitor of Geostationary Meteorological Satellite (GMS), located at about $140^{\circ}$ E. From the GSM data between August 1978 and August 1998, we selected 29 storm events, by examining the $D_{s t}$ index variations. Figure 1 shows two examples from the 29 storms, where the logarithms of electron flux, $D_{s t} \Sigma D_{s t}$ $A L$, and $\Sigma A L$ are plotted from top to bottom, respectively. For the $A L$ and $\Sigma A L$, we used the hourly average $A L$ index.

Figure 1a shows that the electron flux reaches a maximum of more than $10^{4}$ count $\mathrm{cm}^{-2} \mathrm{sec}^{-1} \mathrm{sr}^{-1} \mathrm{MeV}^{-1}$ about 2 days after the $D_{s t}$ minimum. In Fig. 1b, the electron flux about 2 days after the $D_{s t}$ minimum is still low. When we compare $D_{s t}, \Sigma D_{s t} A L$, and $\Sigma A L$ in the region of the flux maximum for Fig. 1a with those for Fig. 1b, it is evident that there is a significant difference. In Fig. 1b, $A L$ does not exceed $500 \mathrm{nT}$ after the $D_{s t}$ minimum, whereas for Fig. 1a, such large values of $A L$ occur several times. Consequently, the magnitude of
$\Sigma A L$ between the two sets of conditions is rather different: in Fig. 1a, the magnitude of $\Sigma A L$ about 2 days after the $D_{s t}$ minimum (at the solid vertical line) is more than three times as large as its magnitude at the same time in Fig. 1b. These facts suggest that $A L$ and $\Sigma A L$ serve as proxies for factors that influence the peak electron flux.

For each storm event, we examined the peak electron flux for the six-day period after the $D_{s t}$ minimum, and the corresponding magnitude of $\Sigma A L$. Figure 2 shows scatter plots of the electron flux (logarithmic scale) against the magnitude of $\Sigma A L$, for all 29 storm cases. Although $\log$ flux values greater than 4 occur for a wide range of $|\Sigma A L|$ (from 18000 to 43000 ), the electron flux tends to be large with increasing $|\Sigma A L|$. The correlation coefficient (CC) is calculated to be 0.64 . This rough correlation supports the proposition that $\Sigma A L$ may be a measure of the control factors in determining the peak electron flux. We also checked if $\Sigma D_{s t}$ shows a similar correlation, but a clear relation was not identified $(\mathrm{CC}=0.11)$. 


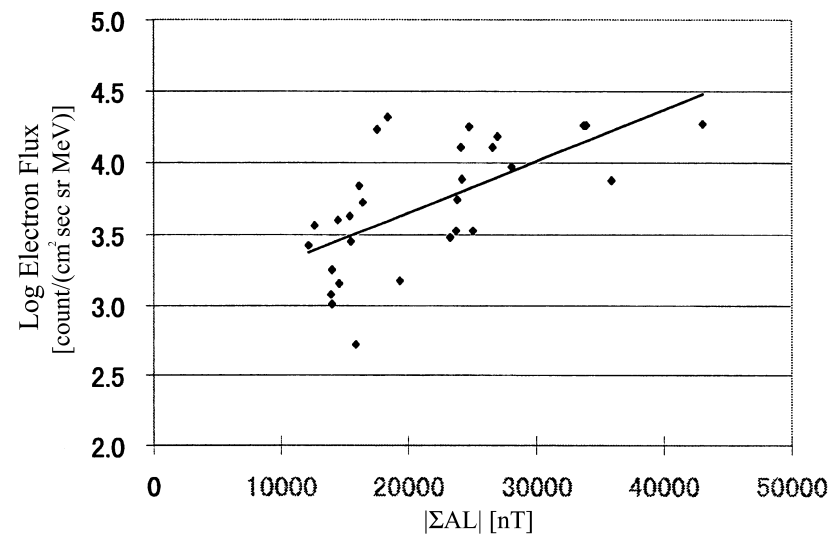

Fig. 2. Scatter plots of the peak electron flux versus the absolute value of $\Sigma A L$ for all 29 events. The solid line indicates the leastsquares fit.

\section{ANN model construction and performance}

In order to construct an ANN model for the relativistic electron flux, we adopted $A L, \Sigma A L, D_{s t} \Sigma D_{s t}$, and UT (= LT $-9 \mathrm{~h}$ for GMS) as input parameters. The $D_{s t}$ index is known to be one of the important parameters that determines the maximum electron flux (e.g. Reeves, 1998). The importance of the history of $D_{s t}$ has been suggested by Stringer et al. (1996), and Freeman et al. (1998), although simple comparison between $\Sigma D_{s t}$ and the peak electron flux does not necessarily identify a clear relation between them. UT is used in the form of $\sin (\mathrm{UT} / 24)$, in order to reproduce a daily variation (or LT dependent structure) of the flux. The recent history of the five parameters was fed to the model. We used an Elman ANN, which is known as a two-layer back-propagation network with feedback connections from the hidden layer to the input layer (Elman, 1990; Wu and Lundstedt, 1997; Kugblenu et al., 2001). The hidden layer has the context neurons, as well as the true input neurons, and the context neurons act as an integrator of temporal information dating back to its initial state; i.e. this gives the network a nonlinear memory capacity.

For the data set for the network training, we selected nine of the 29 storm events in such a manner that the training data set could have various types of flux variations. The nine events consisted of storms on 28 August 1978, 1 January 1980, 6 February 1980, 3 November 1985, 29 November 1985, 4 June 1993, 4 November 1993, 4 April 1994, and 17 April 1994. A total of $1043 \mathrm{~h}$ from these nine events was used for the training. As the learning parameters for training, we chose an RMS error goal of 0.01, an initial adaptive learning ratio of 0.001 , an increasing rate-of-learning ratio of 1.05 , a decreasing rate-of-learning ratio of 0.7 , a momentum constant of 0.95 , an error ratio of 1.04 , and used 5000 learning cycles.

First, we constructed the model for one-hour forward prediction. The performance of the model was then evaluated by comparing the predictions with the observations for the

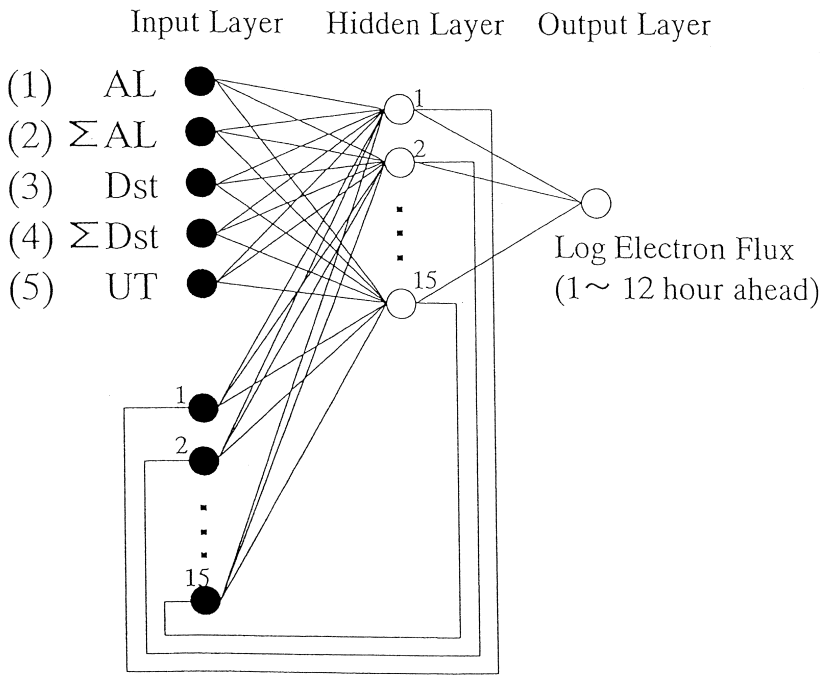

Fig. 3. Architecture of the optimized Elman neural network.

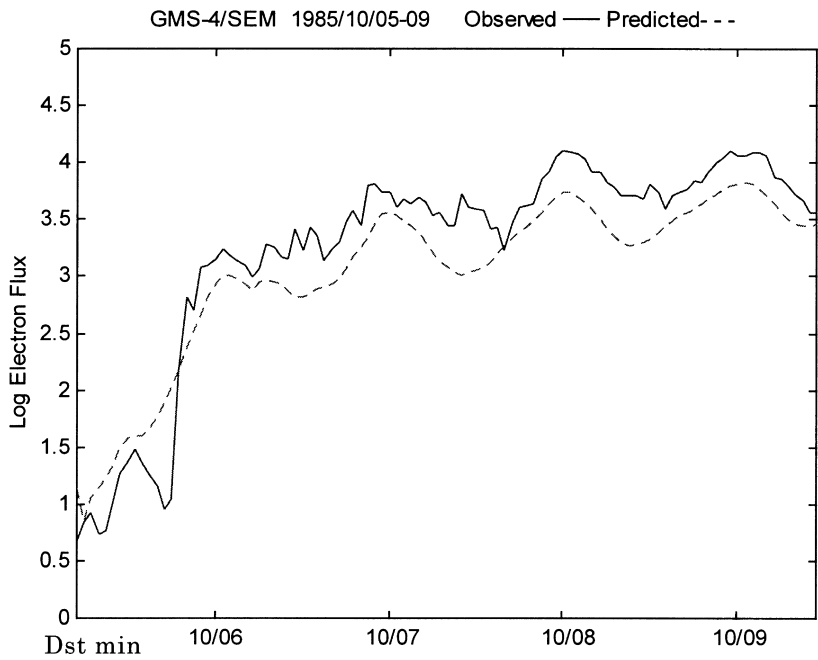

Fig. 4. The storm of 5-9 Oct 1985 for which the best predictability was obtained. The solid and dashed curves represent the observation and one-hour forward prediction, respectively.

remaining 20 storm events ( $2146 \mathrm{~h}$ ). It was found that the optimized ANN had the design shown in Fig. 3, i.e. the number of hidden neurons was 15. This ANN model produced both the highest average value of CC (between the predicted and observed variations), and the highest prediction efficiency (PE) for the 20 test cases. The averages values for $\mathrm{CC}$ and PE were 0.84 and 0.71 , respectively. The average of the RMS error was 0.47 . Figure 4 shows a storm for which this ANN model produced the highest CC (0.97) and PE (0.86) of all the 20 cases. The prediction (dashed curve) follows the observation (solid curve) reasonably well, although the model does not capture the steepness of the rise in flux on 5 October.

We also made some other training data sets by adding several storms to the nine events, and checked the ANN model's performance. The increase in the number of the training data sets did not necessarily improve the prediction performance 


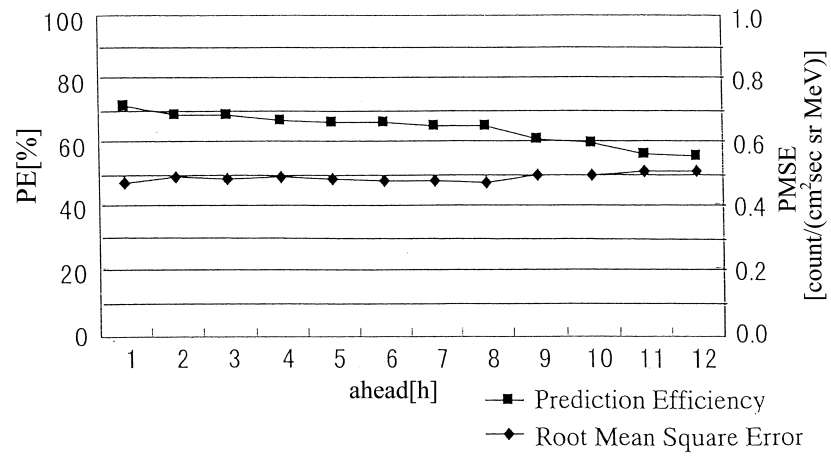

Fig. 5. Change of prediction efficiency (PE) and root-mean-square error (RMSE) for the period 1 to $12 \mathrm{~h}$ ahead.

of the model. However, when we reduced the number of the training data sets, the performance became worse. Although this may be dependent on which storms are included in the training data sets, it is likely that the selected nine events for the training cover various types of flux enhancements.

To identify which input parameters were the most crucial, we dropped $D_{s t}$ and $A L$ from the input parameters, and designed a new ANN. In this model, PE was approximately 0.71 , the same as for the original model. Then, we also dropped $\Sigma D_{s t}$ from the inputs, i.e. the model had only two inputs, $\Sigma A L$ and UT. For this model, PE was about 0.68 , and still close to the original value, indicating that the effect of $\Sigma D_{s t}$ is not very strong. Next, we dropped $\Sigma A L$ instead of $D_{s t}$, i.e. two input parameters, $\Sigma D_{s t}$, and UT, were used. For this model, the prediction efficiency became 0.47 , which is much lower than the original efficiency of 0.71 . We also designed a model with only the $\Sigma A L$ index as the input parameter, i.e. no UT input. The prediction efficiency was 0.64 , which is just below the value of 0.68 that was obtained using the model with $\Sigma A L$ and UT as inputs. From these results, $\Sigma A L$ proved to be the most crucial factor for the fluxenhancement. It should be noted, however, that this $\Sigma A L$ is the quantity after $D_{s t}$ minimum, by which storm times were identified in the first place.

Using the ANN model with inputs of $\Sigma A L, \Sigma D_{s t}$, and UT, we attempted to predict the electron fluxes for the period 2 to $12 \mathrm{~h}$ ahead. The results are summarized in Fig. 5. Although PE gradually decreased from 0.71 as the prediction time increased, it was 0.60 or more for the predictions up to $9 \mathrm{~h}$. Similar ratios and trends were obtained with the five-input model.

If real-time $D_{s t}$ and $A L$ are available, our model can be used for real-time operation. For this, it should be noted that the model starts to produce outputs several hours after the time of $D_{s t}$ minimum, since it is not until $D_{s t}$ recovers to a certain degree that we can determine the time of $D_{s t}$ minimum. However, this delay does not cause a significant problem for the flux prediction, since the flux usually reaches a maximum much later than such a short period of time.

Using a neural network, Koons and Gorney (1991) have shown that up to 10 days of inputs are required to model accurately the electron flux. Our model is not inconsistent with their result. Our model includes $\Sigma D_{s t}$ and $\Sigma A L$ as inputs. Although neural network feedback of our model is a one-hour step (Fig. 3), these two parameters can have the history of $D_{s t}$ and $A L$ from the time of $D_{s t}$ minimum. The maximum of the electron flux usually occurs a few days or more after $D_{s t}$ minimum, and this means that $D_{s t}$ and $A L$ for several days of inputs are used to model.

\section{Concluding remarks}

We have predicted successfully the electron flux using an Elman recurrent-ANN with inputs of $A L, \Sigma A L, \Sigma D_{s t}, D_{s t}$ and UT, or with inputs of $\Sigma A L, \Sigma D_{s t}$, and UT. The feedback connections of the ANN can model effectively the enhancement of the electron flux as a function of the above input parameters, in particular, $\Sigma A L$. The substorm effect represented by $\Sigma A L$ (i.e. the accumulation effect of $A L$ history after $D_{s t}$ minimum) has a significance influence on the flux enhancement in the recovery phase.

It has been accepted that substorms occur more often during southward IMF than northward IMF (e.g. Kamide et al., 1977), although all substorms are not triggered by southward turnings of IMF (e.g. Rostoker, 1983; Lyons et al., 1997). This may appear to indicate that $\Sigma A L$ effects simply reflect the accumulation effect of southward IMF. It should be noted, however, that $|A L|$ tends to be large with the increase in solar wind speed, as well as with the increase in the southward IMF component (e.g. Maezawa and Murayama, 1986). They showed that velocity dependence of the $A L$ index is steeper than linear, approximately proportional to $V^{2}$. Hence, our result that the substorm effect represented by $\Sigma A L$ has a significance influence on the flux enhancement is not inconsistent with the result obtained by Paulikas and Blake (1979), who showed that there is a good correlation between the solar wind velocity and the $\mathrm{MeV}$ electron flux. Some recent papers (e.g. Li et al., 2001) also have indicated the significance of the solar wind speed for the electron flux.

Several researchers (e.g. Baker et al., 1997; Nakamura et al., 1998; Obara et al., 2000b) have suggested that during the main phase, the inward transport of intermediate-energy electrons occurs, and that seed electrons form. This mainphase transport process may be related to substorm activity, such as substorm injections. Our results suggest that as far as the flux level in the recovery phase is concerned, such a substorm process in the main phase of the storm is not important. Rather, recurring substorms after the storm maximum, or the related magnetospheric state, determine to what extent the electrons, which presumably formed in the main phase, are energized.

Acknowledgements. Assistance of computational aspects by H. Nishimura is gratefully acknowledged. The relativistic electron flux data and $D_{s t} / A L$ indices are provided from the Meteorological Satellite Center, Japan, and WDC-C2 of Kyoto University, Japan, respectively. This study was partly supported by the joint research 
program of the Solar-Terrestrial Environment Laboratory, Nagoya University.

Topical Editor C. Chanteur thanks H. Koons and another referee for their help in evaluating this paper.

\section{References}

Baker, D. N., Li, X., Turner, N., et al.: Recurrent geomagnetic storms and relativistic electron enhancements in the outer magnetosphere: ISTP coordinated measurements, J. Geophys. Res., 102, 14 141, 1997.

Elman, J. L.: Finding structure in time, Cognitive Sci., 14, 179, 1990.

Freeman, J. W., O'Brien, T. P., Chan, A. A., and Wolf, R. A.: Energetic electrons at geostationary orbit during the November 34,1993 storm: Spatial/temporal morphology, characterization by a power law spectrum and, representation by an artificial neural network, J. Geophys. Res., 103, 26 251, 1998.

Gussenhoven, M. S., Mullen, E. G., Brautingam, D. H., Holeman, E., and Jorda, C.: Preliminary comparison of dose measurements on CRESS to NASA model predictions, IEEE Trans. Nucl. Sci., 38, 1655, 1991.

Kamide, Y., Perreault, P. D. , Akasofu, S.-I., and Winningham, J. D.: Dependence of substorm occurrence probability on the interplanetary magnetic field and on the size of the auroral oval, J. Geophys. Res., 82, 5521, 1977.

Koons, H. C. and Gorney, D. J.: A neural network model of the relativistic electron flux at geosynchronous orbit, J. Geophys. Res., 96, 5549, 1991

Kugblenu, S., Taguchi, S., and Okuzawa, T.: Consequences of the neural network investigation for $D_{s t}-A L$ relationship, Earth Planets Space, 53, 207, 2001

Li, X., Baker, D. N., Temerin, M., Larson, D., Lin, R. P., Reeves, G. D., Looper, M. D., Kanekal, S. G., and Mewaldt, R. A.: Are energetic electrons in the solar wind the source of the outer radiation belt?, Geophys. Res. Lett., 24, 923, 1997.

Li, X., Temerin, M., Baker, D. N., Reeves, G. D., and Larson, D.: Quantitative prediction of radiation belt elecrons at geostationary orbit based on solar wind measurements, Geophys. Res. Lett., 28, $1887,2001$.
Lyons, L. R., Blanchad, G. T., Samson, J. C., Lepping, R. P., Yamamoto, T., and Moretto, T.: Coordinated observations demonstrating external substorm triggering, J. Geophys. Res., 102, 27 039, 1997.

Maezawa, K. and Murayama, T.: Solar wind velocity effects on the auroral zone magnetic disturbances, in: Solar WindMagnetosphere Coupling, (Eds) Kamide, Y. and Slavin, J. A. pp. 59-83, Terra Scientific Publishing Company, Tokyo, 1986.

Nagai, T.: "Space weather forecast": prediction of relativistic electron intensity at synchronous orbit, Geophys. Res. Lett., 15, 425, 1988.

Nakamura, R., Kamei, K., Kamide, Y., Baker, D. N., Blake, J. B. and Looper, M.: SAMPEX observation of storm-associated electron flux variation in the outer radiation belt, J. Geophys. Res. 103, $26261,1998$.

Obara, T., Nagatsuma, T., Den, M., Sagawa, E., and Onsager, T. G.: Effects of the IMF and substorms on the rapid enhancement of relativistic electrons in the outer radiation belt during storm recovery phase, Adv. Space Res., 26, 89, 2000a.

Obara, T., Nagatsuma, T., Den, M., Miyoshi, Y., and Morioka, A.: Main-phase creation of "seed" electrons in the outer radiation belt, Earth, Planets and Space, 52, 41, 2000b.

Paulikas, G. A. and Blake, J. B.: Effects of the solar wind on magnetospheric dynamics: Energetic electrons at the synchronous orbit, in: Quantitative Modeling of the Magnetospheric Processes, (Ed) Olson, W. P., Geophys. Mongr. Ser., vol. 21, pp. 180-202, AGU, Washington D. C., 1979.

Reeves, G. D.: Relativistic electrons and magnetic storms: 19921995, Geophys. Res. Lett., 25, 1817, 1998.

Rostoker, G.: Triggering of expansion phase intensifications of magnetospheric substorms by northward turnings of the interplanetary magnetic field, J. Geophys. Res., 88, 6981, 1983.

Stringer, G. A., Heuten, I., Salazar, C., and Stokes, B.: Artificial neural network (ANN) forecasting of energetic electrons at geosynchronous orbit, in: Radiation Belts: Models and Standards, (Eds) Lemaire, J. F., Heynderich, D., and Baker, D. N., Geophys. Mongr. Ser., vol. 97, pp. 291-295, AGU, Washington, D. C., 1996.

$\mathrm{Wu}, \mathrm{J}$. and Lundstedt, H.: Geomagnetic storm predictions from solar wind data with the use of dynamic neural networks, J. Geophys. Res., 102, 14 255, 1997. 